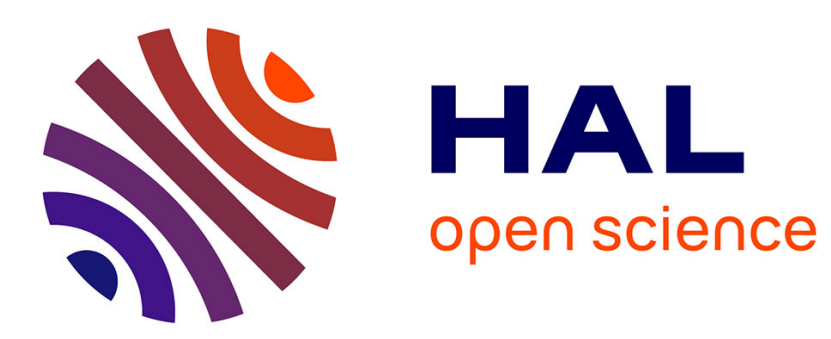

\title{
Computational study of adsorption and the vibrational properties of water on the $\mathrm{Cu}(110)$ surface
}

Lauri Halonen, Elina Sälli, Jukka-Pekka Jalkanen, Kari Laasonen

\section{To cite this version:}

Lauri Halonen, Elina Sälli, Jukka-Pekka Jalkanen, Kari Laasonen. Computational study of adsorption and the vibrational properties of water on the $\mathrm{Cu}(110)$ surface. Molecular Physics, 2007, 105 (09), pp.1271-1282. 10.1080/00268970701420516 . hal-00513104

\section{HAL Id: hal-00513104 \\ https://hal.science/hal-00513104}

Submitted on 1 Sep 2010

HAL is a multi-disciplinary open access archive for the deposit and dissemination of scientific research documents, whether they are published or not. The documents may come from teaching and research institutions in France or abroad, or from public or private research centers.
L'archive ouverte pluridisciplinaire HAL, est destinée au dépôt et à la diffusion de documents scientifiques de niveau recherche, publiés ou non, émanant des établissements d'enseignement et de recherche français ou étrangers, des laboratoires publics ou privés. 


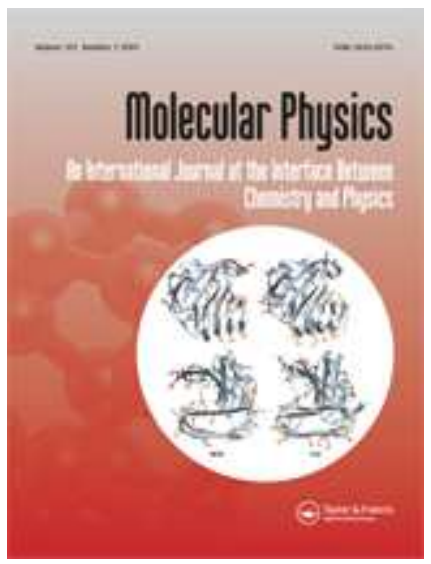

\section{Computational study of adsorption and the vibrational properties of water on the $\mathrm{Cu}(110)$ surface}

\begin{tabular}{|r|l|}
\hline Journal: & Molecular Physics \\
\hline Manuscript ID: & TMPH-2007-0006.R2 \\
\hline Manuscript Type: & Full Paper \\
\hline Author: & 23-Apr-2007 \\
\hline Complete List of Authors: & $\begin{array}{l}\text { Halonen, Lauri; University of Helsinki } \\
\text { Sälli, Elina; University of Helsinki, Laboratory of Physical Chemistry } \\
\text { Jalkanen, Jukka-Pekka; University of Helsinki } \\
\text { Laasonen, Kari; University of Oulu }\end{array}$ \\
\hline Keywords: & adsorption, vibrational spectra, variational calculations \\
\hline & \\
\hline $\begin{array}{l}\text { Note: The following files were submitted by the author for peer review, but cannot be converted } \\
\text { to PDF. You must view these files (e.g. movies) online. }\end{array}$ \\
\hline artvesi10.tex \\
\hline
\end{tabular}

\section{s ScholarONE \\ Manuscript Central}


Computational study of adsorption and the vibrational properties of water on the $\mathrm{Cu}(110)$ surface

\author{
ELINA SÄLLI ${ }^{1}$, JUKKA-PEKKA JALKANEN ${ }^{2}$, KARI LAASONEN ${ }^{3}$, \\ and LAURI HALONEN ${ }^{1,4}$ \\ Laboratory of Physical Chemistry, P.O. Box 55, FIN-00014 University of \\ Helsinki, Finland \\ Department of Chemistry, P.O. Box 3000, FIN-90014 University of Oulu, \\ Finland
}

\footnotetext{
${ }^{1}$ University of Helsinki

${ }^{2}$ Finnish Meteorological Institute, P.O. Box 503 FIN-00101 Helsinki, Finland

${ }^{3}$ University of Oulu

${ }^{4}$ Corresponding author. Email: lauri.halonen@helsinki.fi
} 


\begin{abstract}
This work describes adsorption and internal vibrational motion of the water monomer on the $\mathrm{Cu}(110)$ surface. We have computed the vibrational wave numbers for the adsorbed water using anharmonic variational calculations previously been applied to the gas phase molecule. The three-dimensional potential energy surface for the vibrational motion of the water molecule has been computed using density functional theory (DFT) with periodic boundary conditions, generalized gradient approximation (GGA), plane wave basis sets and projector augmented wave (PAW) potentials as implemented in the Vienna ab initio simulation package (VASP). The data points on the potential energy surface have been fitted into an analytical model and the eigenvalues of the resulting Hamiltonian have been computed variationally.
\end{abstract}

KEYWORDS: adsorption, vibrational spectra, variational calculations 


\section{Introduction}

Vibrational spectra of molecules on the surfaces may be used to identify adsorbed species, distinguish between dissociated, clustered and molecularly adsorbed molecule or study adsorption sites and geometries of the molecule [1], [2]. In addition, the diffusion of molecules, the formation of clusters [3] and surface reactions [4] are assumed to be enhanced by vibrational excitation.

This work describes adsorption of water monomer on the $\mathrm{Cu}(110)$ surface. We chose the surface plane (110) to represent the copper surface due to the following reasons:

i The (110) plane is the roughest of the experimentally most studied copper planes (100), (110) and (111) and thus the interaction of water with $\mathrm{Cu}(110)$ is stronger than with the flatter $\mathrm{Cu}(111)$ or $\mathrm{Cu}(100)$ surfaces.

ii There are experimental data on the vibrational properties of water on the $\mathrm{Cu}(110)$ surface.

According to UPS experiments [5], water bonds to the $\mathrm{Cu}(110)$ surface through the oxygen lone pair which is in agreement with the work function change $-1.0 \mathrm{eV}[7]$. Adsorption of water through the oxygen end has also been observed in Electron Stimulated Desorption (ESD) study [6] where $\mathrm{H}^{+}$ was the only ion detected. A comparison [8] between the dipole moment values of the gas phase and the adsorbed water molecule reveals that water uses only one of its free electron pairs for bonding to the metal surface. This is in agreement with UPS results [5] that can be explained if the symmetry 
axis of water is nearly parallel to the surface. Andersson [9] et al. calculate a tilting angle between the symmetry plane of the water molecule and the normal of the surface to be $57^{\circ}$ on the $\mathrm{Cu}(100)$ surface based on the intensities of the bending mode and the combined translation-rotation mode in the Electron Energy Loss Spectroscopy (EELS) spectra. Based on the polarization selection rules, Mariani and Horn [10] claim that the adsorption system of water on the $\mathrm{Cu}(110)$ surface possesses no symmetry. The bond lengths and bond angle of the water molecule are believed to increase a little in adsorption because the vibrational frequencies of adsorbed water decrease in comparison to the values of the gas phase water molecule [8]. Experimental desorption temperatures of water on the clean $\mathrm{Cu}(110)$ surface vary from $160 \mathrm{~K}$ to $180 \mathrm{~K}$ [11]. Estimates of the adsorption energy or activation energy for desorption vary from -0.67 to $-0.41 \mathrm{eV}$ depending on the model used to interpret desorption temperatures.

The vibrational band positions of the water molecule adsorbed on the $\mathrm{Cu}(110)$ surface have been studied by High Resolution Electron Energy Loss Spectroscopy (HREELS) [8], [12]. A weak bending mode is observed at $1600 \mathrm{~cm}^{-1}$ and a weak broad $\mathrm{OH}$ band around $3370 \mathrm{~cm}^{-1}$ at low coverages. The full-width at half maximum of the stretching band is $400-$ $450 \mathrm{~cm}^{-1}$. It can be separated into three components, $3180 \mathrm{~cm}^{-1}, 3375 \mathrm{~cm}^{-1}$ and $3600 \mathrm{~cm}^{-1}$ when the coverage is increased. These are believed to correspond to the $\mathrm{OH}$ stretching bands of $\mathrm{OH}$-bonds hydrogen bonded to the surface, hydrogen bonded to other water molecules and free, respectively. The bending mode is observed at $1589 \mathrm{~cm}^{-1}[9], 1600 \mathrm{~cm}^{-1}$ [13] and $1630 \mathrm{~cm}^{-1}$ [14] on the $\mathrm{Cu}(100)$ surface. The $\mathrm{OH}$ stretching region is observed around 
$3360 \mathrm{~cm}^{-1}$ [14]. On the other hand, a structureless $\mathrm{OH}$-stretching region between $3230-4030 \mathrm{~cm}^{-1}$ has also been reported when the intensity of the stretching modes has been below the detection limit [9].

The development of electronic structure calculations has made it possible to computationally study detailed properties of molecules adsorbed on metal surfaces. Ruuska et al. [15] have studied the water monomer on the $\mathrm{Cu}(111)$ surface with ten or eight-atomic clusters at the Möller-Plesset (MP2)-theory level using atomic centered basis sets. The atop site was energetically most favorable when full-optimization with a ten-atomic cluster was performed. When a larger 18-atomic cluster model was employed, it was observed that without the Counter Poise (CP) -correction for the Basis Set Superposition Error (BSSE) the orientation, where the hydrogen atoms were parallel to the surface, was energetically the most favorable with adsorption energy $-0.49 \mathrm{eV}$. With the CP correction, the orientation with hydrogens pointing towards the surface was the most favorable with adsorption energy significantly lower, $-0.16 \mathrm{eV}$. Ignaczak et al. [16] used cluster models that contain 2 to 12 atoms. Their density functional (DFT) study showed that the atop site with tilted geometry and tilting angle $50-65^{\circ}$ and the bridge site with upright geometry are energetically almost equivalent on the $\mathrm{Cu}(100)$ surface with adsorption energies around $-0.35 \mathrm{eV}$. They do not mention whether the BSSE correction has been included. Wang et al. [17] modeled the copper planes (100), (110) and (111) with an 18-atomic cluster and studied adsorption energetics and geometries with DFT. Computed non-BSSE corrected adsorption energies are $-0.61 \mathrm{eV}$ for $\mathrm{Cu}(111),-0.81 \mathrm{eV}$ for $\mathrm{Cu}(100)$ and $-1.00 \mathrm{eV}$ for $\mathrm{Cu}(110)$. These values are large compared with experimental 
adsorption energies and other computed values.

Periodic boundary conditions with DFT and generalized gradient approximation (GGA) have been used on copper planes (111) [18], [21], (100) [19], [21] and (110) [20], [21]. All these studied predict the atop site to be the most favorable one with adsorption energies from $-0.22 \mathrm{eV}$ to $-1.11 \mathrm{eV}$. All adsorption geometries are tilted. The $\mathrm{OH}$-bonds of water are slightly elongated and the bond angles are slightly increased from the gas phase values on the $\mathrm{Cu}(100)$ and $\mathrm{Cu}(111)$ surfaces. However, a bond angle as large as $111^{\circ}$ has been reported [20] on the $\mathrm{Cu}(110)$ surface. The results for the $\mathrm{Cu}(100), \mathrm{Cu}(110)[21]$ and $\mathrm{Cu}(111)$ surfaces are similar but the adsorption energies and the bond angles of adsorbed water on the $\mathrm{Cu}(110)$ surface [20] are significantly different. Vibrational frequencies have been calculated on the $\mathrm{Cu}(100)$ surface [22]. In this ab initio work, the surface is modeled with a five-atomic cluster model and normal mode analysis is used to obtain the harmonic vibrational frequencies.

The aim of this computational work is to study adsorption energetics of the water monomer on the copper surface, find the preferred adsorption site and adsorption geometry of the molecule, explain how water bounds to the surface and model the vibrational motion of the molecule on the surface. Our variational approach is based on constructing the quantum mechanical Hamiltonian for the internal vibrational motion and solving the corresponding Schrödinger equation for the vibrational motion. The method is originally developed for gas phase molecules but as far as we know the model with all internal vibrational modes included has not been previously applied to molecules adsorbed on surfaces. In order to keep the number of vibra- 
tional degrees of freedom applicable for the variational approach, we restrict the study to a monomeric water molecule and its internal vibrational modes.

We want to answer the following questions: How does the surface influence the vibrational spectra of the molecule compared to a gas phase molecule? How does the adsorption site and adsorption geometry of the molecule affect the vibrational spectra of the molecule? Is there a correspondence between the interaction mechanism between of the adsorption system and the vibrational wave numbers?

\section{Theoretical and computational model}

\subsection{Vibrational analysis}

The vibrational potential energy surface is obtained from electronic structure calculations. This three dimensional vibrational function has been determined by calculating energies on the discrete data points that describe the change of one coordinate or the simultaneous change of two coordinates from the equilibrium geometry. Calculated data points are fitted into an analytical model as explained in chapter 2.3. For the kinetic energy operator, we have used the exact kinetic energy operator for the gas phase water molecule with the equilibrium geometry of the adsorbed molecule. Vibrational kinetic energy operator of the gas phase water molecule is [23]

$$
\hat{T}_{v}=-\frac{\hbar^{2}}{2} \sum_{i j}^{3}\left(\frac{\partial}{\partial q_{i}}+\frac{1}{J} \frac{\partial J}{\partial q_{i}}\right) g^{\left(q_{i}, q_{j}\right)} \frac{\partial}{\partial q_{j}}
$$

where coordinates $q_{i}$ are displacements from the equilibrium bond length, $\triangle r_{1}, \triangle r_{2}$ and the displacement from the bond angle $\triangle \theta$ and $g$-matrix ele- 
ments are given by

$$
q^{\left(q_{i}, q_{j}\right)}=\sum_{\alpha=1}^{3} \frac{1}{m_{\alpha}}\left(\nabla_{\alpha} q_{i}\right) \cdot\left(\nabla_{\alpha} q_{j}\right)
$$

The volume element of integration needed to calculate matrix elements $\int d \tau \psi \hat{T}_{v} \psi$ and $\int d \tau \psi \hat{V}_{v} \psi$ is

$$
d \tau=J d r_{1} d r_{2} d \theta
$$

where the Jacobian of the coordinate transformation is

$$
J=\left|\operatorname{det} g^{\left(q_{i} q_{j}\right)}\right|^{-\frac{1}{2}} \prod_{\alpha=1}^{3} m_{\alpha}^{-\frac{3}{2}} .
$$

We have applied an appropriate unitary similarity transformation to the kinetic energy operator in order to have a unit weight factor $J$ in the volume element [23]. The g-matrix elements are presented, for example, in reference [24]. The eigenvalues of the vibrational Hamiltonian have been obtained variationally.

The use of the exact kinetic energy operator is based on the assumption that the surface perturbs the potential energy surface of the molecule and its equilibrium geometry only. In practice, the metal surface perturbs the adsorbed molecule and changes its translational and rotational degrees of freedom into low-frequency vibrations that are called frustrated translations and rotations [1]. In addition, the phonon modes of the metal surface may couple to other vibrational modes. On the $\mathrm{Cu}(110)$ surface, the hindered translation and libration modes have been observed at 460 and $745 \mathrm{~cm}^{-1}$ by HREELS [8], [12]. These values are low compared with high-frequency modes of water as the bending mode at $1600 \mathrm{~cm}^{-1}$ is the lowest of them. We assume that the ordinary vibrational modes of the molecule are uncoupled to 
the translational and rotational vibrations. Therefore, we can use the exact gas phase kinetic energy operator for the adsorbed molecule. Our assumption is justified by the difference of these wave numbers [25]. The same adiabatic assumption has previously been applied to ammonia adsorbed on the $\mathrm{Ni}(111)$ surface [26] and tested in the case of the possible coupling of the $\mathrm{N}-\mathrm{Ni}$ stretching and $\mathrm{N}-\mathrm{H}$ stretching.

\subsection{Electronic structure calculations}

Electronic structure calculations were required for relaxations of the isolated water molecule, the copper(110) surface and the water-surface system. All calculations were performed using Vasp (Vienna ab initio simulation package) [27]. It is a code for electronic structure calculations and molecular dynamics simulations in solid state materials but it can also be applied to surface calculations with a slab model.

Calculations were based on the density functional theory (DFT) with the generalized gradient approximation (GGA) of Perdew and Wang (PW91), PAW pseudo potentials [28] and plane wave basis sets as implemented in Vasp. The energy cut-off for plane waves was $520 \mathrm{eV}$. Fermi level was smeared by the 2nd order Methfessel-Paxton method with a width of $0.4 \mathrm{eV}$. All calculations were spin restricted. Electronic energy was converged to at least $10^{-4} \mathrm{eV}$ in all calculations. For the $k$-point sampling of the Brillouin zone for integrations, the Monkhorst-Pack $k$-mesh 441 (16 irreducible $k$-points) was employed for relaxations when we tried to find the most favorable adsorption site and geometry for the adsorbed water molecule. A denser Monkhorst-Pack $k$-mesh 661 (36 irreducible $k$-points) was chosen for 
the final relaxations of the water-surface system.

Modeling of the gas phase water molecule needed to be done with artificial periodicity because of the plane wave code employed. The isolated water molecule was placed inside a cubic super cell with dimensions of $10 \AA$. Only one $k$-point (the gamma point) was used in integrations because the periodicity was artificial. The copper(110) surface was modeled with the slab model: five layers of metal atoms with four atoms in each layer and a vacuum region of $10 \AA$ between the layers. A theoretical lattice constant value of $3.63 \AA$ obtained in our calculations was used instead of the experimental one $3.61 \AA[29]$. The reconstruction of the bare surface decreased the lattice constant in the direction of the surface normal close to the surface by $9 \%$ compared with the calculated bulk lattice constant. The adsorbed molecule was placed on one side of the slab and periodic boundary conditions were used to multiply the super cell in three dimensions as shown in figure 1 .

Conjugate gradient and quasi-Newton methods were employed in the structural searches. The water molecule and the two uppermost layers of the copper surface were relaxed while the bottom layers were fixed at their bulk positions until the Hellman Feynman forces on all relaxed atoms were less than $0.05 \mathrm{eV}^{-1}$. A stricter value $0.005 \mathrm{eV}^{-1}$ was used in the final relaxations.

For each discrete data point on the potential energy surface of the internal coordinates of the water molecule, only the degrees of freedom that correspond the studied part of the potential energy surface were changed. The metal surface and the coordinates of the water molecule with respect to the surface were fixed at their equilibrium positions. We found that the 
definition of the bending motion was not obvious. There is a unique definition of the bending coordinate for a gas phase molecule [30]. For a molecule adsorbed on the surface, this coordinate is mixed with coordinates that describe hindered rotations with respect to the surface. We decided to test this by computing the total energies of the asymmetrically adsorbed molecule by performing bending by moving one of the hydrogens only, moving the other hydrogen atom only and moving both hydrogens equally so that the approximate direction of the symmetry axis of the water molecule was kept fixed. The effect of the choice of the bending coordinate was found insignificant. Eventually, we defined the bending coordinate to satisfy the latter condition.

\subsection{The potential energy surface}

Morse coordinates $y_{i}=1-e^{-a \triangle r_{i}}$ were used as coordinates for the stretches instead of displacement coordinates $\Delta r_{i}=r_{i}-r_{i}(e q)$ from the equilibrium bond length $r_{i}(e q)$. Direct displacement coordinates $\triangle \theta=\theta-\theta(e q)$ from the equilibrium bond angle $\theta(e q)$ were used for the bending. The potential energy function used is similar to one in Ref [31]. In all the fits, the potential energy parameters were optimized with the non-linear least-square method using MATHEMATICA.

Twelve data points from -0.4 to $1.2 \AA$ on the stretching part of the potential were fitted into a potential energy model

$$
D_{2} y_{1}^{2}+D_{3} y_{1}^{3}+D_{4} y_{1}^{4}
$$

where we denote parameters for one OH-bond by $D_{2}, D_{3}$ and $D_{4}$ and $D_{2}^{\prime}, D_{3}^{\prime}$ and $D_{4}^{\prime}$ for the other one. The value of the Morse parameter $a\left(a^{\prime}\right.$ for the other one) obtained was used as a parameter in the following fits. For the isolated 
water molecule, the symmetric and upright adsorption geometry, only one stretching curve was computed because the bonds are identical, whereas for the asymmetric adsorption geometry the data points were computed for both bonds and two sets of parameters were optimized.

The bending curve was determined using 13 data points in the range $50^{\circ}<\theta<180^{\circ}$ and the points were fitted into the potential energy function

$$
C+\frac{1}{2} f_{\theta \theta} \triangle \theta^{2}+\frac{1}{6} f_{\theta \theta \theta} \triangle \theta^{3}+\frac{1}{24} f_{\theta \theta \theta \theta} \triangle \theta^{4}
$$

where $C$ and $f_{i}$ are parameters.

The potential energy coupling between two bond oscillators was calculated using 16 data points from $-0.2 \AA$ to $0.2 \AA$ and the potential energy coupling between the bending and stretching motions was calculated with 16 points from $-0.2 \AA$ to $0.2 \AA$ and $90^{\circ}$ to $120^{\circ}$. The energies were fitted into potential energy function

$$
E\left(\triangle r_{1}, \triangle r_{2}, 0\right)=E\left(\triangle r_{1}, 0,0\right)+E\left(0, \triangle r_{2}, 0\right)+E(0,0,0)+\left(a a^{\prime}\right)^{-1} f_{r r^{\prime}} y_{1} y_{2}
$$

where a harmonic force constant $f_{r r^{\prime}}$ describes strength of the interaction. By $E\left(\triangle r_{1}, \triangle r_{2}, \triangle \theta\right)$ we denote energy of a data point with stretches $\triangle r_{1}$ and $\triangle r_{1}$ and bond angle displacement $\triangle \theta$. The coupling points of bending and stretching were fitted into analytical model

$$
\begin{gathered}
E\left(\triangle r_{1}, 0, \triangle \theta\right)=E\left(\triangle r_{1}, 0,0\right)+E(0,0, \triangle \theta)+E(0,0,0)+a^{-1} f_{r \theta} y_{1} \triangle \theta \\
+\frac{1}{2} a^{-1} f_{r \theta \theta} y_{1} \triangle \theta^{2}+\frac{1}{2}\left(a^{-2} f_{r r \theta}+a^{-1} f_{r \theta}\right) y_{1}^{2} \triangle \theta
\end{gathered}
$$

where $f_{i}$ force constants describe the strength of the bend-stretch interaction.

The effect of changing the bond angle and the bond lengths at the same time was tested and found to have such a small effect on the vibrational wave 
numbers that the appropriate potential energy terms were were omitted from this work.

\subsection{Variational calculations}

Vibrational wave numbers were obtained variationally with a program originally written for the symmetric water molecule and modified for this problem where the water molecule possesses asymmetric structure. The matrix elements were calculated either with analytical formulas or numerically with Gauss-Hermite and Gauss-Laquerre numerical integration methods. See ref. [31] and [32] for more details. Products of harmonic oscillator eigenfunctions for the bend and Morse oscillator eigenfunctions for the stretches were used as basis functions. All the bending and stretching states up to seven quanta were included. The convergence with respect to the to the number of integration points and the size of the basis set was tested in the case of the gas phase water using variational program for the symmetric water where more basis functions were available in the program. A convergence better than $0.1 \mathrm{~cm}^{-1}$ was achieved for all reported transitions.

\section{Results and discussion}

\subsection{Convergence tests}

Convergence tests were performed with respect to the size of the $k$-point mesh, cut-off energy, the width of the Fermi level smearing, spin polarization and the size of the super cell for the most favorable symmetric adsorption geometry. Results showed that convergence with respect to the adsorption 
energy is obtained within $3 \%$ and with respect to the structural parameters at least within $8 \%$. The $k$-point mesh 881 (32 non-reduced points) and 661 (18 non-reduced points) gave identical results excluding tilting angle whereas there is a small numerical difference between the $k$-point mesh 441 (8 non-reduced $k$-points) and 661 . The difference was small and therefore the $k$-point mesh 441 was used in finding the adsorption geometry and 661 only for final relaxations. Cut-off energy $800 \mathrm{eV}$ gave the same results as the cut-off energy $520 \mathrm{eV}$. Spin-polarization had no effect on the geometry and only small effect on adsorption energy. However, the energy difference was too small to require more expensive spin-unrestricted calculations. We found out that the size of the super cell has clearly more effect on the adsorption geometry and energetics than any of the computational parameter. Results of the convergence tests with respect to the size of the super cell are presented in table 1.

Although $2 \times 2$ and $3 \times 3$ slabs with coverages 0.25 and 0.11 gave acceptable adsorption energies, we also studied the possibility of direct lateral interactions between water molecules by computing energies of water molecules inside the super cells that had been used in surface calculations without the surface. We used $k$-point mesh 661 instead of a gamma point for $k$-space integrations to allow the interaction between neighboring cells. Energy difference was $0.002 \mathrm{eV}$. 


\subsection{Adsorption energetics and mechanism of water on} the $\mathrm{Cu}(110)$ surface

There are four adsorption sites of high symmetry on the (110) surface of the fcc metal : an atop site right above a copper atom of the first layer, large and short bridge sites between the copper atoms of the first layer and a hollow site above a copper atom on a second layer. In addition, the direction of copper rows defines symmetric geometries on these sites yielding ten symmetric adsorption geometries.

We calculated first adsorption energy and geometry on the symmetric adsorption geometries. We employed an assumption that water bounds to the surface through the oxygen atom as suggested by [33]. However, all the coordinates of the water molecule were allowed to relax. It was found that the most favorable adsorption site is atop and the adsorption geometry is tilted with respect to the surface. Then we performed a few tests with asymmetric geometries and found out that a lower adsorption energy was achieved when adsorption geometry is asymmetric. We continued with non-symmetric adsorption geometries and tested eleven non-equivalent asymmetric initial adsorption geometries. We chose these initial geometries so that the oxygen atom was on the atop site and the bond lengths and the bond angle of water corresponded to the gas phase values of water. The resulting geometries were such that either both hydrogens point away from the surface or one hydrogen points away from the surface and the other is almost parallel to the surface or directed to the surface. Eight of these eleven initial geometries produced an asymmetric adsorption geometry that was energetically most favorable. Finally we performed relaxations with convergence criteria $0.005 \mathrm{eV}^{-1}$ for 
forces and a $k$-point mesh 661 for this most favorable asymmetric adsorption geometry and the most favorable symmetric adsorption geometry.

The computational adsorption energy is defined as $E_{a}=E_{r e l}\left(\mathrm{H}_{2} \mathrm{O}+\mathrm{Cu}\right)-$ $E_{\text {rel }}\left(\mathrm{H}_{2} \mathrm{O}\right)-E_{\text {rel }}(\mathrm{Cu})$, where $E_{\text {rel }}\left(\mathrm{H}_{2} \mathrm{O}+\mathrm{Cu}\right), E_{\text {rel }}\left(\mathrm{H}_{2} \mathrm{O}\right)$ and $E_{\text {rel }}(\mathrm{Cu})$ are the energies of the relaxed water-copper system, relaxed isolated water molecule and relaxed copper surface, respectively. Symmetric and asymmetric adsorption geometries are almost equivalent energetically although the asymmetric geometry is slightly more favorable with an adsorption energy of $-0.36 \mathrm{eV}$ than the symmetric adsorption geometry that possesses an adsorption energy of $-0.35 \mathrm{eV}$. Experimental adsorption energies vary from $-0.67 \mathrm{eV}$ to $-0.41 \mathrm{eV}$. These numbers are somewhat larger than our values. There could be at least two explanations for that: (i) It is known that DFT is not capable of describing the dispersion forces that might play a role in the interaction between the water molecule and the surface. (ii) The experimental desorption temperatures and thus experimental adsorption energies correspond to activation energies for desorption and not necessarily adsorption energies.

It is common for these structures that the adsorption site is atop. There is a small lateral displacement of the oxygen atom from the exact atop site in the case of the symmetric and asymmetric adsorption geometry. That is also ascertained on the $\mathrm{Cu}(100)$ and $\mathrm{Cu}(111)$ surfaces [19], [18]. We assumed initially that water bounds to the surface through the oxygen atom which was also confirmed in all of our calculations. The distance between oxygen and the atop metal atom is $2.2 \AA$ for the symmetric geometry and $2.1 \AA$ for the asymmetric geometry. The most favorable adsorption geometries are tilted with respect to the surface in agreement with experiments [8], [9] 
and [5] and almost all computational results. The tilting angle is $71^{\circ}$ for the symmetric adsorption geometry and $80^{\circ}$ for the asymmetric adsorption geometry. One of the OH-bonds is directed almost along the 'long' copper row in the asymmetric geometry and there is no symmetry with respect to the surface. The associated hydrogen atom directs to the surface. The other bond directs away from the surface. In the symmetric adsorption geometry, the water molecule and the surface possess one common symmetry plane and both hydrogens point away from the surface. We found out that the most favorable adsorption geometry is asymmetric with respect to the surface in agreement with Mariani and Horn [10]. The calculated bond lengths $0.976-$ $0.981 \AA$ and bond angle $105.2-105.3^{\circ}$ of water on both adsorption geometries are slightly increased compared to the calculated gas phase values of $0.971 \AA$ and $104.6^{\circ}$. Experimental values for the gas phase water are $0.957 \AA$ and $104.5^{\circ}$, respectively [36].

For comparison, we also performed relaxations for the most favorable upright adsorption geometry on the atop site. In this upright geometry, the molecule is perpendicular with respect to the surface and all the atoms of the molecule are right above the long copper row. The bond lengths $0.973 \AA$ are slightly shorter than in the case of other adsorption geometries but there is a significant increase in the bond angle which is $108.5^{\circ}$. The distance between the surface and the oxygen atom is $2.18 \AA$. The interaction between the water molecule in the upright adsorption geometry and the surface is weaker as the adsorption energy is only $-0.27 \mathrm{eV}$. All the structures are presented in figure 2 and our best geometrical parameters for water on the $\mathrm{Cu}(110)$ surface are presented in the Table 1 . The asymmetric structure is similar 
to the one obtained earlier in a work where a DFT method with periodic boundary conditions were also employed [21].

The bonding of the water monomer to the metal surfaces is usually explained by the interaction between the dipole moment of the water molecule and its induced dipole moment beneath the metal surface and the overlapping of the d-orbitals of the metal surface and the lone pair orbitals of the water molecule [1], [2]. The adsorption energy as small as $-0.36 \mathrm{eV}$ in absolute value indicates physisorption interaction. However, the tilted adsorption geometry points out to the formation of a chemical bound between the molecule and the surface because upright geometry has been claimed to enhance the dipole induced dipole interaction [18]. The $1 \mathrm{~b}_{1}$ and $3 \mathrm{a}_{1}$ orbitals of the gas phase water molecule are believed to be involved in bonding with the surface whereas the $2 \mathrm{a}_{1}$ and $1 \mathrm{~b}_{2}$ orbitals do not affect the bonding. The $3 \mathrm{a}_{1}$ orbital is in the symmetry plane of the molecule whereas the $1 b_{1}$ orbital is orthogonal to the plane of the molecule. [2] Based on the symmetry arguments, interaction with the surface through the $3 \mathrm{a}_{1}$ orbital is enhanced in the upright adsorption geometry whereas the interaction through the $1 b_{1}$ orbital favors the parallel geometry. Previous works on other copper planes [19], [18] give similar results.

In order to analyze the interaction mechanism of the water molecule with the copper surface, we computed the density of the states (DOS) for the relaxed copper surface without the water molecule and for the symmetric and asymmetric water-surface systems. For comparison, we also computed the DOS for the most favorable upright adsorption geometry. To analyze the observed changes in the DOS curves, we computed the local density of 
the states (LDOS) of the adsorbed water molecule in symmetric, asymmetric and upright adsorption geometry and compared them with the density of the states of the gas phase molecule. We also computed the local densities of the states of the s, p and d orbitals of the copper atoms of the adsorption system and compared them with the corresponding LDOS of the bare metal slab. $[34]$

The DOS for the bare metal surface and the symmetric adsorption system are presented in figure 3 . There are changes in regions around $-22.5 \mathrm{eV}$, $-10.3 \mathrm{eV}$ and $-6.9 \mathrm{eV}$ due to adsorption as indicated with arrows. Analysis of the LDOS of the symmetric adsorption system revealed that the observed new peaks in figure $3 \mathrm{~B}$ correspond to the $2 \mathrm{a}_{1}, 1 \mathrm{~b}_{2}$ and $3 \mathrm{a}_{1}$ orbitals of the water molecule. The peak that corresponds to the $1 b_{1}$ orbital of the gas phase water molecule was found to be hidden under the states of copper in the region around $-5.1 \mathrm{eV}$. The DOS and LDOS figures of asymmetric adsorption geometry are similar to the symmetric case expect the $1 b_{2}$ orbital where a small change is observed. Clear differences can be seen on the DOS and LDOS curves for upright adsorption geometry when compared with the other adsorption geometries.

To analyze the differences between the flat and upright adsorption geometries we used the energy of the $2 a_{1}$ orbital as the zero-point energy of the systems because according to the literature the energetic position of the $2 a_{1}$ orbital of the water molecule is not affected in the adsorption [1]. The LDOS for symmetric, asymmetric and upright adsorption geometry are presented in figure 4 . The vertical solid lines indicate approximate positions of the orbitals of gas phase water obtained in our calculations. In the upright 
geometry, the $1 b_{1}$ orbital is shifted $0.5 \mathrm{eV}$ less and $3 a_{1} 0.5 \mathrm{eV}$ more from the computed values for the gas phase water than the flat geometries. The deviation in the positions and the heights of the peaks indicates differences in the bonding mechanism between flat and upright adsorption systems. For comparison, we have experimental positions of experimental UPS spectra peaks from references [10], [5] and [35]. They report the energetic positions of the $1 b_{2}, 3 a_{1}$ and $1 b_{1}$ orbitals only and because of that we chose to compare the energetic differences of the experimental and computed peaks instead of direct energies of the orbitals. Computed differences in the LDOS peaks and the experimental ones are tabulated in the table 3 where fairly good agreement of our calculated values with experimental values is seen.

As seen from the figure $3 \mathrm{a}$, the bare copper surface possesses no states around the $2 \mathrm{a}_{1}$ and $1 \mathrm{~b}_{2}$ states of water. Thus, these orbitals do not significantly affect the bonding between the molecule and the metal surface. It is common for all adsorption systems that the $1 b_{2}$ orbital remains practically unchanged but the $1 b_{1}$ and $3 a_{1}$ orbitals are shifted to lower energies and the LDOS peaks of the adsorption systems are usually smaller in energies than the peaks of the gas phase water molecule. The LDOS of symmetric and asymmetric adsorption geometries are almost identical and the energetic position and the height of the $1 b_{1}$ orbital peak change most. The $3 \mathrm{a}_{1}$ orbital change most with the upright adsorption geometry. This indicates that the flat adsorption geometry enhances interaction between the $1 b_{1}$ orbital and the upright geometry enhances interaction with the $3 \mathrm{a}_{1}$ orbital as expected due to symmetry arguments. In addition, the analysis of the LDOS projected on the s, p and d orbitals of copper of the bare surface and copper atoms of 
the adsorption systems revealed that the lone pair orbitals of water interact with the d orbitals of the copper surface. Possible interaction with the $\mathrm{p}$ orbitals of the copper surface was not observed.

\subsection{Vibrational analysis}

For consistency, we wanted to use the same potential energy model for all geometries. In order to minimize the number of data point computed for the adsorbed geometries, where even single point energy calculations are time consuming, we performed variational calculations for the gas phase water with different potential energy functions and potential energy parameters obtained with different number of data points. The convergence with respect to different number of data points and terms in the potential energy functions is shown in tabel 4.

We assured that twelve data points in the vicinity of the minimum and a simple potential energy function $D_{2} y^{2}+D_{3} y^{3}+D_{4} y^{4}$ provides a sufficient model. The results for the gas phase water are presented in the table 4 . We decided to use this computationally cheap treatment for the adsorption geometries instead of more complicated models. The bond that directs to the surface in the asymmetric adsorption geometry is an expection. We found out that there is an attractive interaction between the hydrogen atom that directs to the metal surface. For that curve, we computed more data points and also removed some data points where the $\mathrm{H}$ atom is too close to the metal surface and fitted the points with a model with terms from $y^{2}$ to $y^{10}$. The calculated wave numbers for the gas phase water are lower than corresponding experimental values, as expected. See for example ref. [39]. 
The stretching potential energy curves of the isolated water molecule, symmetric, upright and asymmetric adsorption geometry are presented in figure 5. The stretching curves for the bonds in the symmetric and upright adsorption geometry and the bond of the asymmetric adsorption geometry that points away from the surface are identical. A significant lowering of the dissociation energy occurs with the bond of the asymmetric geometry that is directed to the metal surface. The bond is a bit longer than the others and there is an attractive interaction with the copper surface. All the adsorption geometries possess lower dissociation energies than the gas phase molecule. The parameters for the potential energy surfaces are in table 5 and the calculated vibrational wave numbers in table 6 where the experimental wave numbers for the gas phase water are also given.

The wave numbers of the stretching modes of the adsorption geometries are lower than the corresponding gas phase values. The wave numbers of the stretching modes of the upright geometry are closer to the gas phase values than the corresponding wave numbers of the flat geometries due to a weaker interaction with the metal surface. Otherwise, the spectral positions are similar to the symmetric adsorption case. The different interaction mechanism of the symmetric and upright geometry does not seem to have significant influence on the spectral positions as the largest differences are between the wave numbers of the symmetric and asymmetric structure where the interaction mechanisms are similar. This could have been expected beforehand because the stretching motion of the water molecule changes mainly the positions of the hydrogen atoms and has little effect on the lone pair orbitals. The experimental $\mathrm{OH}$ - stretching region of the spectra of the adsorbed water 
molecule is broad with a full width at half maximum of $400-450 \mathrm{~cm}^{-1}$ even at low temperatures. It can be resolved into three components at $3180 \mathrm{~cm}^{-1}$, $3375 \mathrm{~cm}^{-1}$ and $3600 \mathrm{~cm}^{-1}$. They have been explained by three kinds of $\mathrm{OH}-$ bonds: bonded to the surface, hydrogen bonded and free bonds, respectively [8]. All our computed values for the stretching fundamentals are in the middle of the experimental stretching region bands particularly when the expected deviation of $100 \mathrm{~cm}^{-1}$ is taken into account. The surface selection rule [38] implies that only modes with an oscillating dipole perpendicular to the metal surface are infra red (IR) active. The detailed analysis of the intensities of the different transitions would require computing the dipole moment surface of the system, which, in this case, is not a well-defined problem due to the periodic boundary conditions employed to a metallic system. We decided to estimate the intensities of the reported transitions by using the dipole moment transitions vectors for the gas phase water [37] and projecting them on the axis perpendicular to the surface. It was found out that the asymmetric modes of the symmetric and upright geometries are IR inactive, all the symmetric modes and the mode (100) of the asymmetric structure possess similar intensities and the (010) mode of the asymmetric structure is more intensive than the others (see table 6 for labeling the states). Symmetric vibrations of the symmetric and upright geometry correspond to the 'free $\mathrm{OH}$-bond case' reported in experimental works. The interpretation of the vibrations of the asymmetric structure is not evident. The eigenfunctions of the vibrational states showed that the bond directed away from the surface is responsible for the vibration with wave number $3560 \mathrm{~cm}^{-1}$ and the wave number $3347 \mathrm{~cm}^{-1}$ corresponds to the bond directed to the surface. The peak at $3180 \mathrm{~cm}^{-1}$ 
seen in the spectra has previously been assigned to the OH-bond directed to surface. According to our calculations, bonding to the surface decreases the wave number of the stretch but the decrease is not that large. Actually, our computed value is closer to the peak around $3375 \mathrm{~cm}^{-1}$ that according to Lackey corresponds to hydrogen-bonded OH-bonds [8].

The bending mode for adsorbed water has been observed around $1600 \mathrm{~cm}^{-1}$ [8], which is the same value as for the gas phase water molecule. Our computed values are lower than the computed gas phase values by more than $30 \mathrm{~cm}^{-1}$. Hydrogen bonding is possibly seen in the experimental results because according to Thiel [1] the interaction with the surface decreases the wave numbers for bending but the hydrogen bonding increases it. Clustering of the water molecule is assumed to occur on the $\mathrm{Cu}(110)$ surface even if the coverage is small [8]. Bending wave number decrease in adsorption has also been reported by Ribarsky [22], although his harmonic normal mode analysis was based on a five-atomic cluster model.

\section{Conclusions}

We have studied adsorption of water on the $\mathrm{Cu}(110)$ surface using the density functional theory with the slab model. We have found out that water bounds to the atop site with a weak chemisorption bond between the lone pair orbitals of the oxygen atom and the d orbitals of the metal atoms. The most favorable adsorption site is atop and the adsorption geometry is tilted and asymmetric with respect to the surface in agreement with experimental results and previous similar computational works. The calculated orbital energies for the tilted geometries are similar to the experimental ones, which 
also confirms that the adsorption geometry of water is tilted. Adsorption energies are around $-0.36 \mathrm{eV}$. Experimental adsorption energies vary from -0.67 to $-0.41 \mathrm{eV}$ depending on the interpretation of the desorption temperatures.

We have computed vibrational transitions using a model where the Hamiltonian for the vibrational motion have been constructed and its eigenvalues have been computed variationally. The work is based on the adiabatic approximation where there is no coupling of the internal vibrational modes of the molecule with the frustrated translations and rotations or to the phonon modes of the surface. Without the adiatic assumption the computed positions of the spectral peaks for the adsorbed geometries would have splittings which in addition to clustering are seen as broadening in the experimental spectra. In principle, it is possible to compare the wave numbers computed in different adsorption geometries with the experimental spectra and to make conclusions about the possible adsorption geometry of the molecule. This is potentially important when the interpretation of the experimental results is difficult due to broad spectra caused by many simultaneous adsorption geometries or clustering.

Our computed density-functional wave numbers have deviations from the experimental wave numbers more than $100 \mathrm{~cm}^{-1}$ for the stretches and $50 \mathrm{~cm}^{-1}$ for the bending mode. Luckily, the differences between adsorbed geometries and gas phase structures are produced better. The wave numbers of the adsorbed water molecule are lower than the gas phase values which indicates softening of the bonds. It may explain why water is assumed to dissociate on the surface and why it is more reactive when the molecule adsorbs. For 
example, lowering of the dissociation energy is easily seen from the stretching potential energy curves. The interaction mechanism does not have large effect on the vibrational wave numbers. However, when the interaction is the weakest, the wave numbers are closer to experimental values. The bond directed to the metal surface produces lower stretching fundamental wave number than the others which support the experimental assumption that the lowest peak of the stretching region is associated with the bonds directed to the surface.

\section{Acknowledgments}

We would like to thank CSC Scientific Computing Ltd for providing computer time and Magnus Ehrnrooth Foundation for financing the project. Laboratory of Physical Chemistry has been a part of The Center of Excellence in Computational Molecular Science funded by the Academy of Finland and University of Helsinki during part of the work. 


\section{References}

[1] P. A. Thiel, T. E. Madey. Surf. Sci. Rep., 7, 211 (1987).

[2] M. Henderson. Surf. Sci. Rep., 46, 1 (2002).

[3] K. Morgenstern, K. Rieder. J. Chem. Phys., 116, 135746 (2002)

[4] R. Smith, D. Killelea, D. DelSesto, A. Utz. Science, 304, 5673992 (2004).

[5] A. Spitzer, H. Lüth. Surf. Sci., 120, 376 (1988).

[6] M. Polak. Surf. Sci., 321, 249 (1994).

[7] G. Mariani, K. Horn. Surf. Sci., 126, 279 (1983), Bange, K., R. Döhl, D.E. Grider, J.K. Sass. Vacuum, 33, 10757 (1983), K. Bange, D. E. Grider, T. E. Madey, J. K. Sass. Surf. Sci., 136, 38 (1984) and A. Spitzer, H. Lüth. Surf. Sci., 120, 376 (1988).

[8] D. Lackey, J. Schott, B. Straehler, J. Sass. J. Chem. Phys., 91, 21365 (1989)

[9] S. Andersson, C. Nyberg, C. Tengstål. Chem. Phys. Lett., 104, 4305 (1984)

[10] G. Mariani, K. Horn. Surf. Sci., 126, 279 (1983).

[11] D. Lackey, J. Schott, B. Straehler, J. Sass. J. Chem. Phys., 91, 21365 (1989), K. Andersson, A. Gomez, C. Glover, D. Nordlund, H. Öström, T. Schiros, O. Takahashi, H. Ogasawara, L. G. M. Pettersson, A. Nilsson. Surf. Sci. Lett., 585, L183 (2005). Bange, K., R. Döhl, D.E. Grider, J.K. Sass. Vacuum 33, 10757 (1983), K. Bange, D. E. Grider, T. E. Madey, 
J. K. Sass. Surf. Sci., 136, 38 (1984), Electrochim. Acta, 36, 11/12 1883 (1991).

[12] J. Sass, D. Lackey, J. Schott. Electrochim. Acta, 36, 11/12 1883 (1991).

[13] A. Carley, P. Davies, M. Roberts, K. Thomas. Surf. Sci. Lett., 238, L467 (1990).

[14] T. Sueyoshi, T. Sasaki, Y. Iwasawa. J. Phys. Chem. B, 101, 4648 (1997)

[15] H. Ruuska, T. Pakkanen, R. Rowley. J. Chem Phys. B, 108, 132614 (2004).

[16] A. Ignaczak, J. A. N. F. Gomes. J. Electroan. Chem., 420, 209 (1997).

[17] G. C. Wang, L. Jiang, X. Y. Pang, Z. S. Cai, Y. M. Pan, X. Z Zhao, Y. Morikawa, J. Nakamura. Surf. Sci., 543, 118 (2003).

[18] A. Michaelides, V. A. Ranea, P. L. de Andres, D. A. King. Phys. Rev. Lett., 90, 21216102 (2003).

[19] S. Wang, Y. Cao, P. A. Rikvold. Phys. Rev. B, 70, 205410 (2004).

[20] S. Izvekov, A. Mazzolo, K. VanOpdorp, G. A. Voth. J. Chem. Phys., 114, 73248 (2001).

[21] Q.-L. Tang, Z.-X. Chen, Surf. Sci. doi: 10.1016/j.susc.2006.11.036 (2006).

[22] M. Ribarsky, W. Luedtke, U. Landman. Phys. Rev. B, 32, 21430 (1985).

[23] J. Pesonen, L. Halonen. Adv. Chem. Phys., 125, 269 (2003).

28 
[24] H. Wei, T. Carrington. J. Chem. Phys., 97 5, 3029 (1992).

[25] M. Born, K. Huang. Dynamical theory of Crystal lattices. Oxford at the Clarendon press (1956).

[26] T. Kurten, M. Biczysko, T. Rajamäki, K. Laasonen, L. Halonen. J. Phys. Chem. B, 109 (18), 8954-8960 (2005).

[27] G. Kresse, J. Hafner. Phys. Rev. B, RC558 (1993) and G. Kresse, J. Furthmüller. Comput. Mat. Sci., 6, 15 (1996); Phys. Rev. B, 54, 11169 (1996).

[28] G. Kresse, D. Joubert. Phys. Rev. B, 59, 31758 (1999).

[29] N. Ashcroft, N. Mermin. Solid State Physics, (Saunders College, Philadelphia (1981).

[30] A. R. Hoy, I. M. Mills, G. Strey. Mol. Phys., 24, 1265 (1972).

[31] E. Kauppi, L. Halonen. J. Phys. Chem., 94, 5779 (1990).

[32] A. H. Stroud. Gaussian Guadrature Formulas, Prentice-Hall, Inc, Englewood Cliffs, N.J. (1966).

[33] G. Mariani, K. Horn. Surf. Sci., 126, 279 (1983), D. Lackey, J. Schott, B. Straehler, J. Sass. J. Chem. Phys., 91, 21365 (1989), Bange, K., R. Döhl, D.E. Grider, J.K. Sass. Vacuum, 33, 10757 (1983), K. Bange, D. E. Grider, T. E. Madey, J. K. Sass. Surf. Sci., 136, 38 (1984), A. Spitzer, H. Lüth. Surf. Sci., 120, 376 (1988), M. Polak. Surf. Sci., 321, 249 (1994).

[34] We have used the programs p4V and LEV00 as tools to analyze the charge density and density of the states of the 
molecule. See http://cms.mpi.univie.ac.at/odubay/p4vasp/ and http://www.cmmp.ucl.ac.uk/ lev/codes/lev00/ for details.

[35] Bange, K., R. Döhl, D.E. Grider, J.K. Sass. Vacuum, 33, 10757 (1983).

[36] J. Tennyson, N. F. Zobov, R. Williamson, O. L. Polyansky. J. Phys. Chem., Ref. Data. 30, 3735 (2001).

[37] U. Jørgensen, P. Jensen. J. Mol. Spect., 161, 219 (1993).

[38] N. Sheppard, J. Erkelens. Appl. Spect., 38 4, 471 (1984).

[39] J. Sauer, P. Ugliengo, E. Garrone, R. Saunders. Chem. Rev. 94, 2095 (1994) 


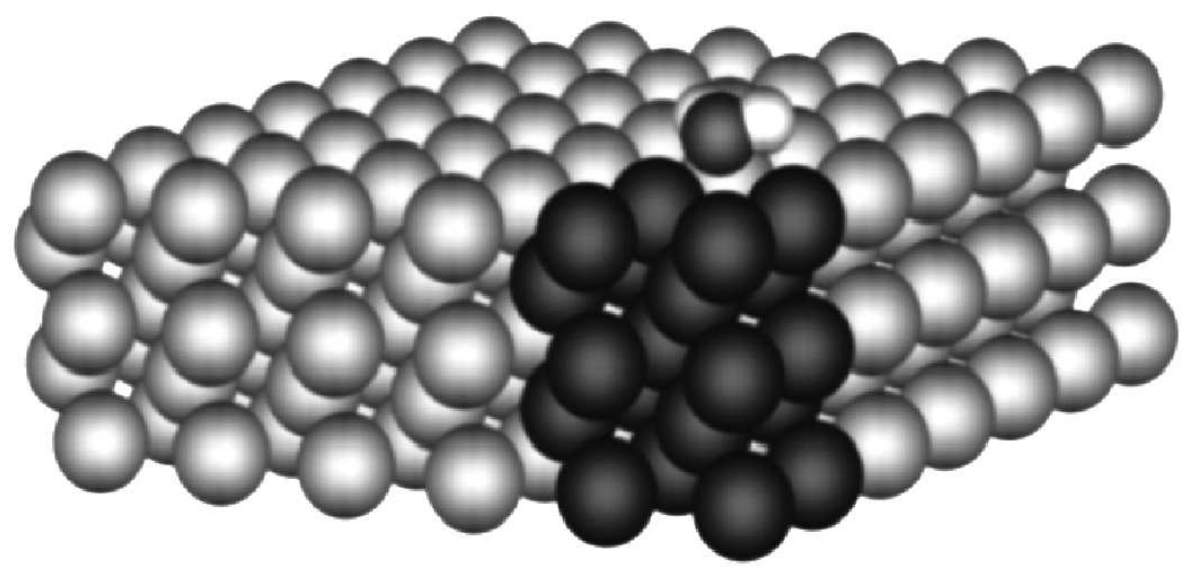

Figure 1: The slab model. Dark atoms represent atoms in one super cell. 


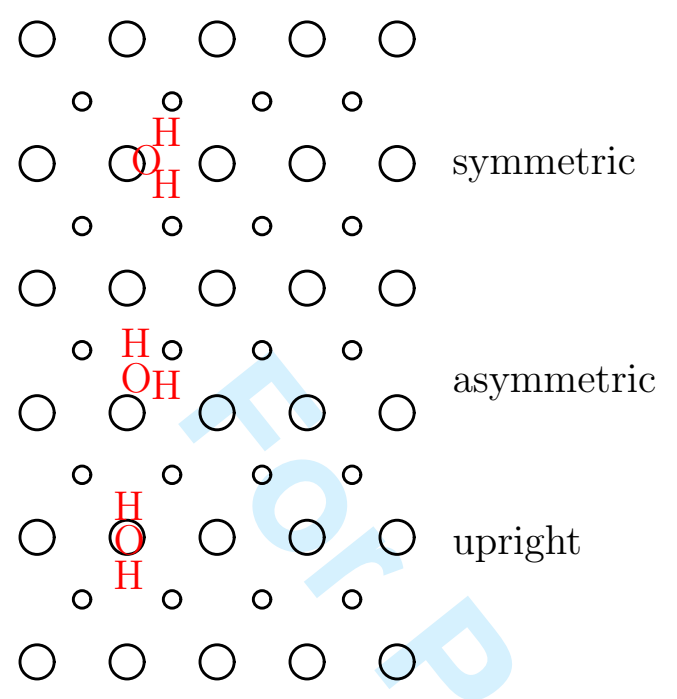

Figure 2: Symmetric, asymmetric and upright adsorption geometry 

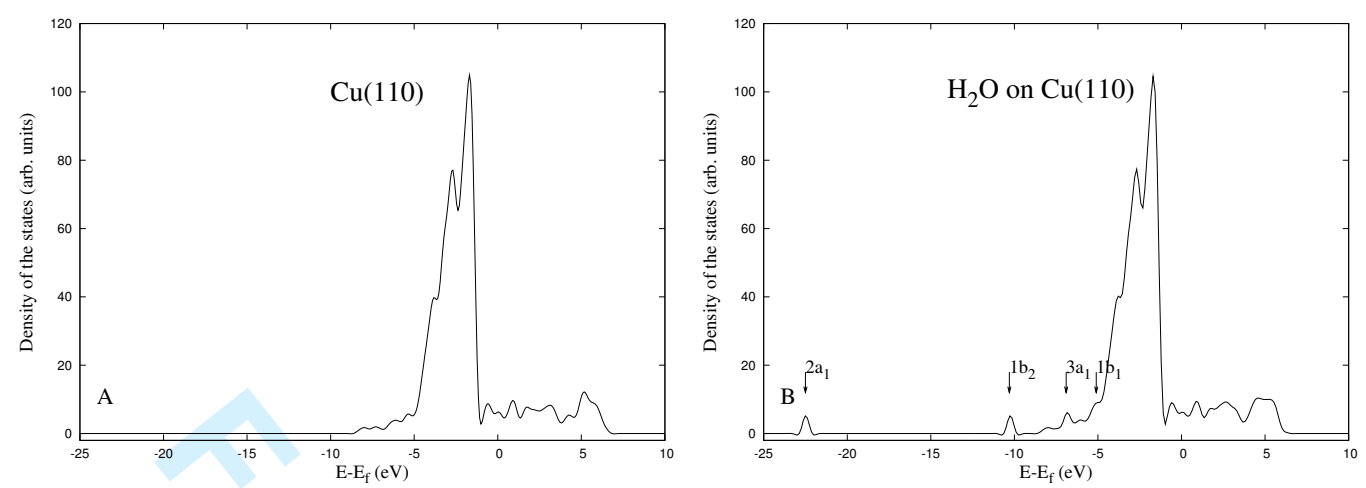

Figure 3: (A) Density of the states (DOS) of the $\mathrm{Cu}(110)$ surface. (B) Density of the states in the symmetric adsorption system of water and the $\mathrm{Cu}(110)$ surface. The arrows point DOS-peaks that are due to adsorption. 


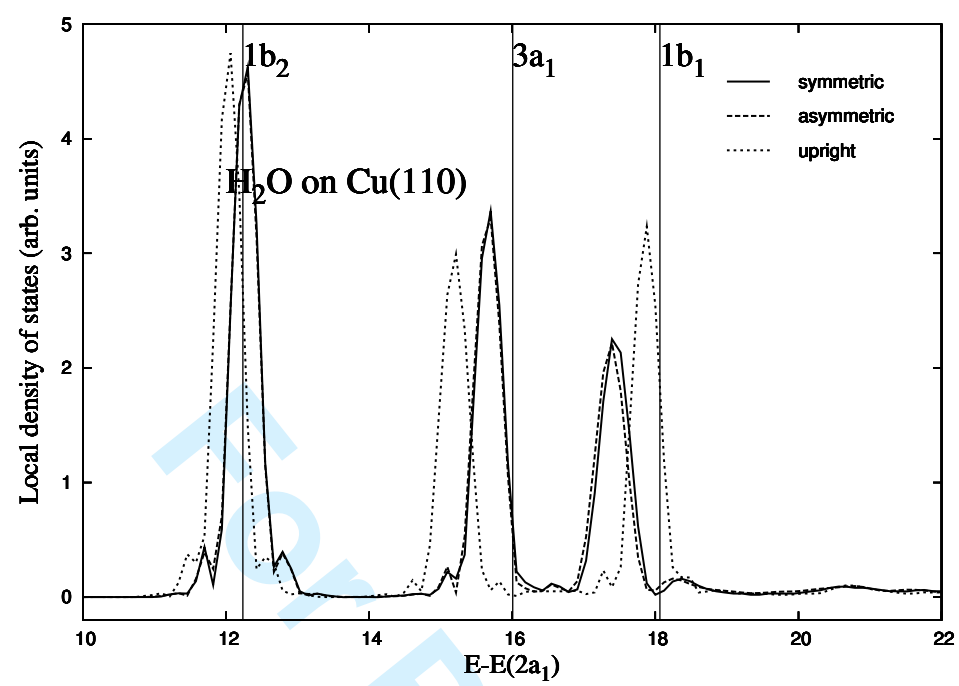

Figure 4: Local density of the states (LDOS) of the symmetric, asymmetric and upright adsorption systems and density of the states of the water molecule. The DOS curves for the symmetric and asymmetric adsorption geometries are almost identical expect for the lowest molecular orbital around $-5 \mathrm{eV}$ where a small difference can be seen. The arrows represent the approximate positions of the molecular orbitals of the gas phase water molecule. Energies are given in eV. The energy axes in this figure is chosen such that the energetic position of the $2 a_{1}$ orbital is set to zero. 


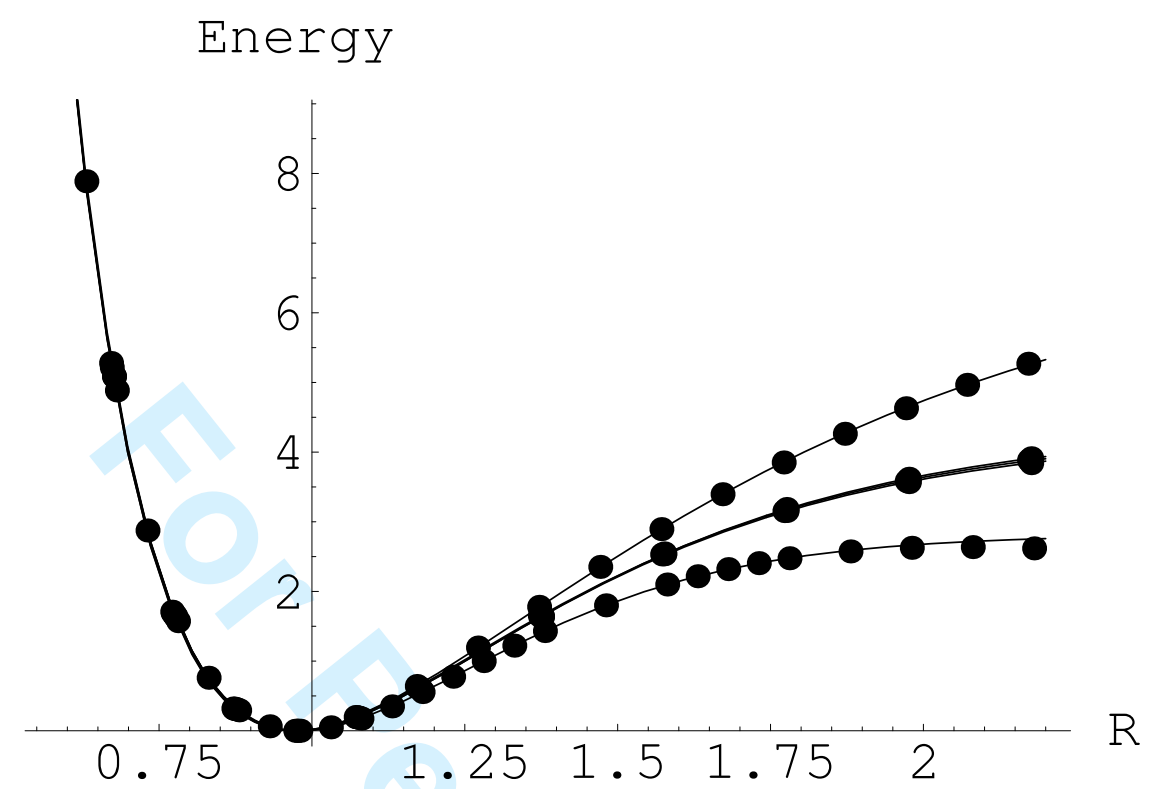

Figure 5: Potential energy curves for stretching. The topmost curve corresponds to the gas phase water. The curves for the symmetric and upright adsorption geometry are one on the other with the the bond of the asymmetric structure that directs away from the surface. The lowest curve corresponds to the bond of the asymmetric structure that is directed to the surface. Energy is given in $\mathrm{eV}$ and the bond length $R$ in $\AA$. 


\begin{tabular}{|c|c|c|c|c|c|}
\hline size of the super cell & $E_{\text {ads }}(\mathrm{eV})$ & $r_{\mathrm{OH}}(\AA)$ & $\theta(\mathrm{deg})$ & $r_{\mathrm{CuO}}(\AA)$ & $\phi(\mathrm{deg})$ \\
\hline $\begin{array}{c}2 \times 2 \text { slab, 5 layers } \\
10 \AA \text { vacuum }\end{array}$ & -0.35 & 0.976 & 105.3 & 2.23 & 71.3 \\
\hline $\begin{array}{c}3 \times 3 \text { slab,5 layers } \\
10 \AA \text { vacuum }\end{array}$ & -0.35 & 0.976 & 105.7 & 2.20 & 65.8 \\
\hline $\begin{array}{c}2 \times 2 \text { slab, } 7 \text { layers } \\
10 \AA \text { vacuum }\end{array}$ & -0.35 & 0.976 & 105.3 & 2.23 & 72.3 \\
\hline $\begin{array}{c}2 \times 2 \text { slab, 9 layers } \\
10 \text { vacuum }\end{array}$ & -0.36 & 0.976 & 105.4 & 2.22 & 71.4 \\
\hline $\begin{array}{c}2 \times 2 \text { slab, } 5 \text { layers } \\
15 \AA \text { vacuum }\end{array}$ & -0.34 & 0.977 & 105.2 & 2.24 & 74.8 \\
\hline
\end{tabular}

Table 1: The result of the convergence test with respect to the size of the super cell 


\begin{tabular}{|c|c|c|c|c|}
\hline & gas phase & symmetric & asymmetric & upright \\
\hline$r_{A}(\AA)$ & 0.971 & 0.976 & 0.976 & 0.973 \\
\hline$r_{B}(\AA)$ & 0.971 & 0.976 & 0.981 & 0.973 \\
\hline$\theta_{e}(\mathrm{deg})$ & 104.6 & 105.3 & 105.2 & 108.5 \\
\hline$r_{\mathrm{CuO}}(\AA)$ & - & 2.23 & 2.14 & 2.18 \\
\hline$\phi(\mathrm{deg})$ & - & 71.3 & 79.7 & 90 \\
\hline$E_{a}(\mathrm{eV})$ & - & -0.35 & -0.36 & -0.27 \\
\hline
\end{tabular}

Table 2: The quantities $r_{A}$ and $r_{B}$ are the bond lengths and $\theta_{e}$ is the bond angle of the water molecule, $r_{\mathrm{CuO}}$ is the distance between the oxygen atom and the copper atom below it, $\phi$ is the angle between the surface normal and the water plane and $E_{a}$ is the adsorption energy as defined in the text. 


\begin{tabular}{|c|c|c|c|c|c|}
\hline & gas phase (exp) & symmetric & asymmetric & upright & exp \\
\hline$E\left(1 b_{1}\right)-E\left(3 a_{1}\right)(\mathrm{eV})$ & $2.1(2.1)$ & 1.7 & 1.8 & 2.7 & $1.6-2.0$ \\
\hline$E\left(3 a_{1}\right)-E\left(1 b_{2}\right)(\mathrm{eV})$ & $3.8(3.9)$ & 3.4 & 3.3 & 3.1 & $3.8-4.2$ \\
\hline
\end{tabular}

Table 3: The separation of the $1 b_{1}$ and $3 a_{1}$ and $3 a_{1}$ and $1 b_{2}$ peaks in computed LDOS curves and corresponding values from references [10], [35] and [5]. Experimental values in the parenthesis have been taken from the reference $[35]$ 


\begin{tabular}{|c|c|c|c|}
\hline number of the data points & 12 & 28 & 28 \\
\hline model & $D_{2} y^{2}+D_{3} y^{3}+D_{4} y^{4}$ & $D_{2} y^{2}+D_{3} y^{3}+D_{4} y^{4}$ & from $y^{2}$ to $y^{10}$ \\
\hline$(00)_{+}+$ & 1545 & 1545 & 1545 \\
\hline$(10)_{+} 0$ & 3555 & 3552 & 3550 \\
\hline$(10)_{-} 0$ & 3649 & 3647 & 3642 \\
\hline
\end{tabular}

Table 4: The convergence with respect to the potential energy surface model and the choice of the parameters. Wave numbers are given in $\mathrm{cm}^{-1}$.

See table 6 for the labeling the states. In the model on the right hand side, the Morse parameter $a$ has been constrained to the value obtained from the model $D_{2} y^{2}+D_{4} y^{4}$ and the data points have then fitted into a model that contains all potential energy terms from $y^{2}$ to $y^{10}$. 


\begin{tabular}{|c|c|c|c|c|}
\hline parameter & gas phase & symmetric & upright & asymmetric \\
\hline$a\left(\AA^{-1}\right)$ & 1.4501 & 2.49258 & 2.42155 & 2.42642 \\
\hline$D_{2}(\mathrm{aJ})$ & 1.4501 & 0.637031 & 0.681347 & 0.671124 \\
\hline$D_{3}(\mathrm{aJ})$ & -0.670345 & -0.0177527 & -0.0146682 & - \\
\hline$D_{4}(\mathrm{aJ})$ & 0.344533 & 0.032307 & 0.0305745 & 0.0329666 \\
\hline$a^{\prime}\left(\AA^{-1}\right)$ & & & & 2.64959147 \\
\hline$D_{2}^{\prime}(\mathrm{aJ})$ & & & & 0.513237 \\
\hline$D_{3}^{\prime}\left(\mathrm{aJ}^{\prime}\right.$ & & & & 0.013466 \\
\hline$D_{4}^{\prime}\left(\mathrm{aJ}^{-2}\right.$ & & & & 0.0282639 \\
\hline$f_{r r^{\prime}}\left(\mathrm{aJ} \AA^{-2}\right)$ & -0.0948686 & -0.0725689 & -0.105353 & -0.0668896 \\
\hline$\left.f_{\theta \theta}(\mathrm{aJ} \mathrm{rad})^{-2}\right)$ & 0.678738 & 0.656309 & 0.612189 & 0.64792 \\
\hline$f_{\theta \theta \theta}\left(\mathrm{aJ} \mathrm{rad}^{-3}\right)$ & -0.846216 & -0.817509 & 0.784897 & -0.8086 \\
\hline$f_{\theta \theta \theta \theta}\left(\mathrm{aJ} \mathrm{rad}^{-4}\right)$ & -0.426945 & -0.39709 & -0.272521 & -0.351787 \\
\hline$f_{r \theta}\left(\mathrm{aJ} \AA^{-1} \mathrm{rad}^{-1}\right)$ & 0.27246 & -0.241484 & 0.233361 & 0.251393 \\
\hline$f_{r \theta \theta}\left(\mathrm{aJ} \AA^{-1} \mathrm{rad}^{-2}\right)$ & -0.24166 & -0.294004 & -0.293499 & -0.267818 \\
\hline$f_{r r \theta}\left(\mathrm{aJ} \AA^{-2} \mathrm{rad}^{-1}\right)$ & -0.234299 & -0.26423 & -0.12719 & -0.235541 \\
\hline$f_{r^{\prime} \theta}\left(\mathrm{aJ} \mathrm{rad}^{-1}\right)$ & & & & 0.192319 \\
\hline$f_{r^{\prime} \theta \theta}\left(\mathrm{aJ} \AA^{-1} \mathrm{rad}^{-2}\right)$ & & & & -0.393742 \\
\hline$f_{r^{\prime} r^{\prime} \theta}\left(\mathrm{aJ} \AA^{-2} \mathrm{rad}^{-1}\right)$ & & & & -0.399861 \\
\hline
\end{tabular}

Table 5: The numerical values of the parameters from the fit of the calculated potential energy points on the potential energy function. The coefficients of terms from $y^{5}$ to $y^{10}$ that were used for the bond directed to the surface in the asymmetric structure are not shown. 


\begin{tabular}{|c|c|c|c|c|c|c|}
\hline local & gas phase & gas phase exp & symmetric & upright & local & asymmetric \\
\hline$(00)_{+} 1$ & 1545 & 1595 & 1514 & 1469 & 001 & 1499 \\
\hline$(00)_{+} 2$ & 3051 & 3152 & 2990 & 2899 & 002 & 2958 \\
\hline$(10)_{+} 0$ & 3555 & 3657 & 3491 & 3507 & 100 & 3347 \\
\hline$(10)_{-} 0$ & 3649 & 3756 & 3578 & 3618 & 010 & 3560 \\
\hline$(10)_{+} 1$ & 5089 & 5235 & 4991 & 4961 & 101 & 4823 \\
\hline$(10)_{+} 1$ & 5185 & 5331 & 5080 & 5075 & 011 & 5046 \\
\hline$(20)_{+} 0$ & 7005 & 7202 & 6822 & 6872 & 200 & 6480 \\
\hline$(20)_{-} 0$ & 7049 & 7250 & 6822 & 6922 & 110 & 6851 \\
\hline$(11)_{+} 0$ & 7236 & 7445 & 7090 & 7161 & 020 & 6959 \\
\hline
\end{tabular}

Table 6: The vibrational transitions of the isolated water monomer and the water monomer adsorbed on the $\mathrm{Cu}(110)$ surface. Experimental values are from reference [36]. All values are given in $\mathrm{cm}^{-1}$. Local mode assignments of the transitions have been used for all the structures. Two numbers in the quantum labels express two $\mathrm{OH}$ bonds and the third number indicates the number of bending quanta. For symmetric structures, we denote symmetric vibration by + and asymmetric vibration by - . 


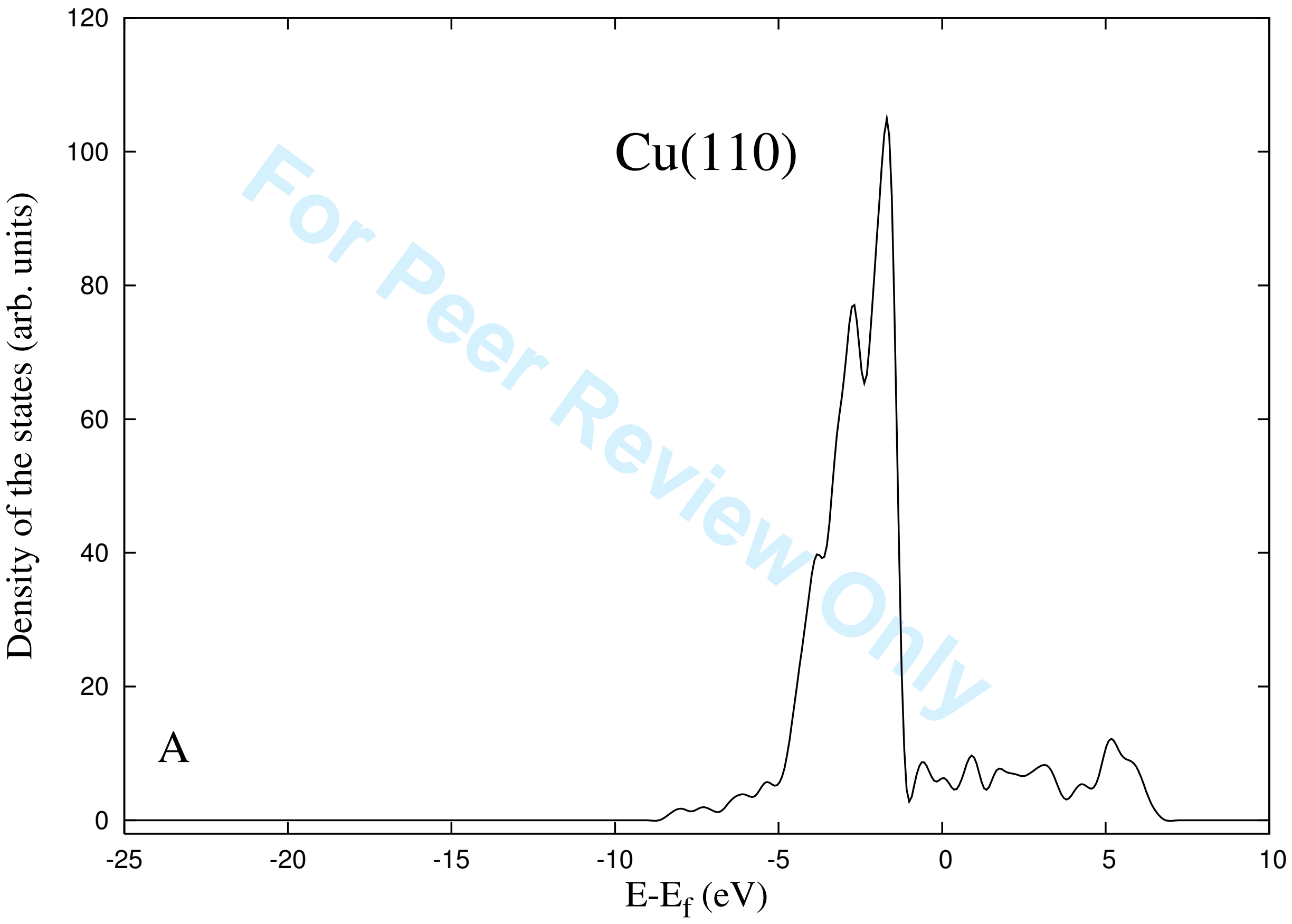

URL: http://mc.manuscriptcentral.com/tandf/tmph 


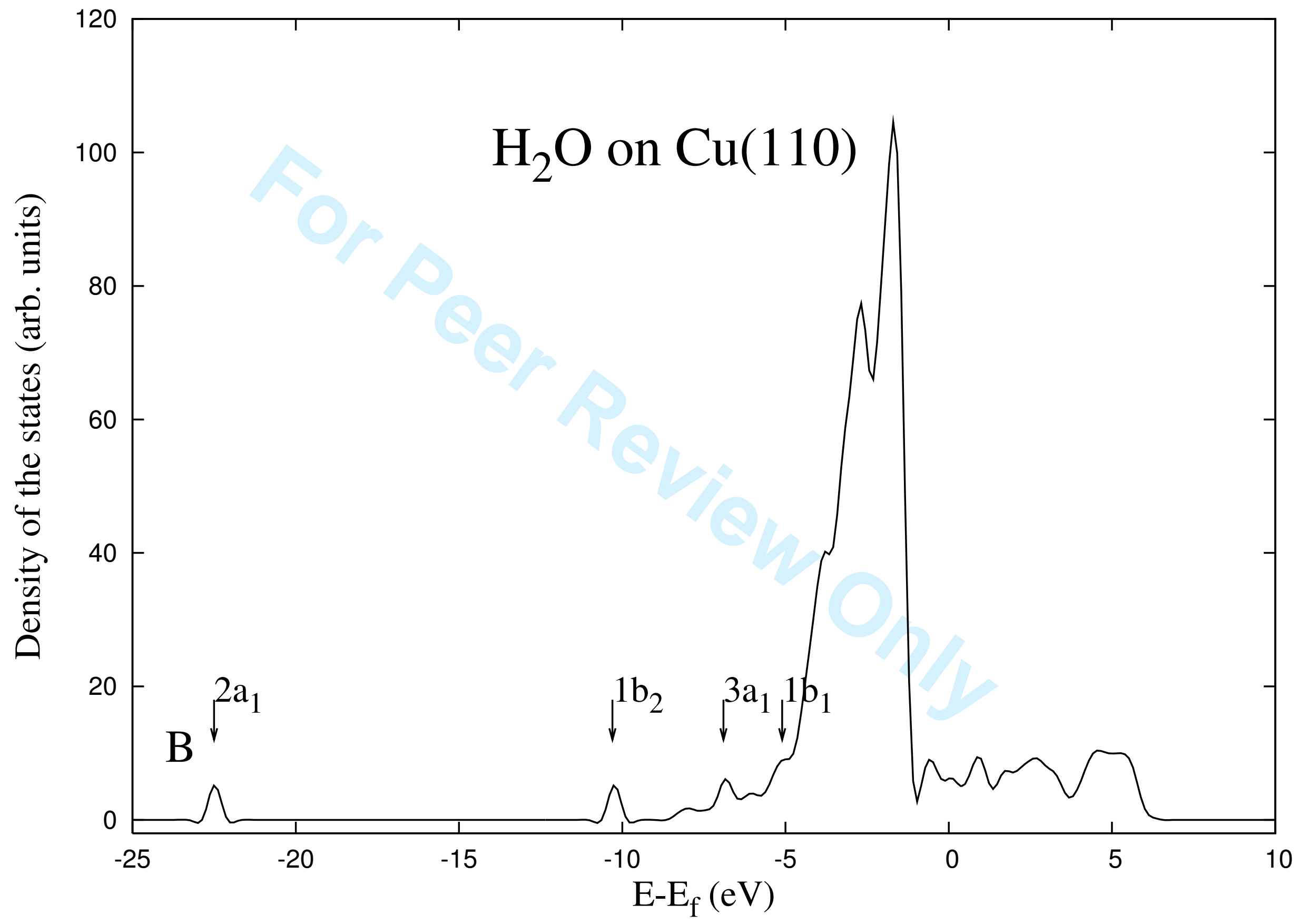




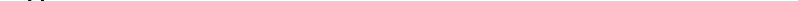


Energy

Page 45 of 46Molecular Physics

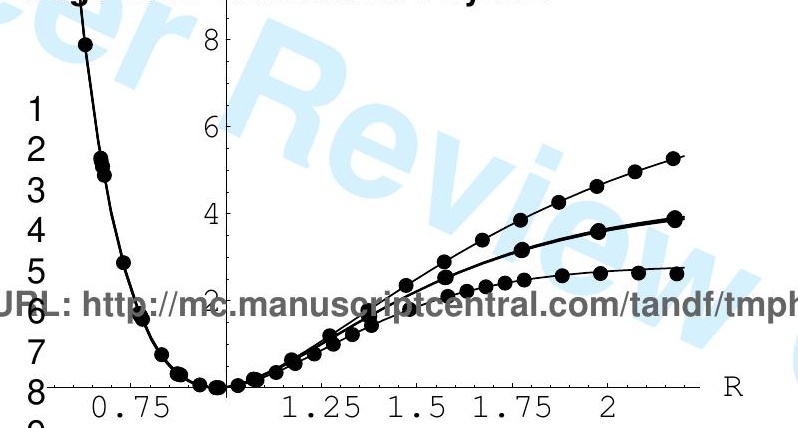




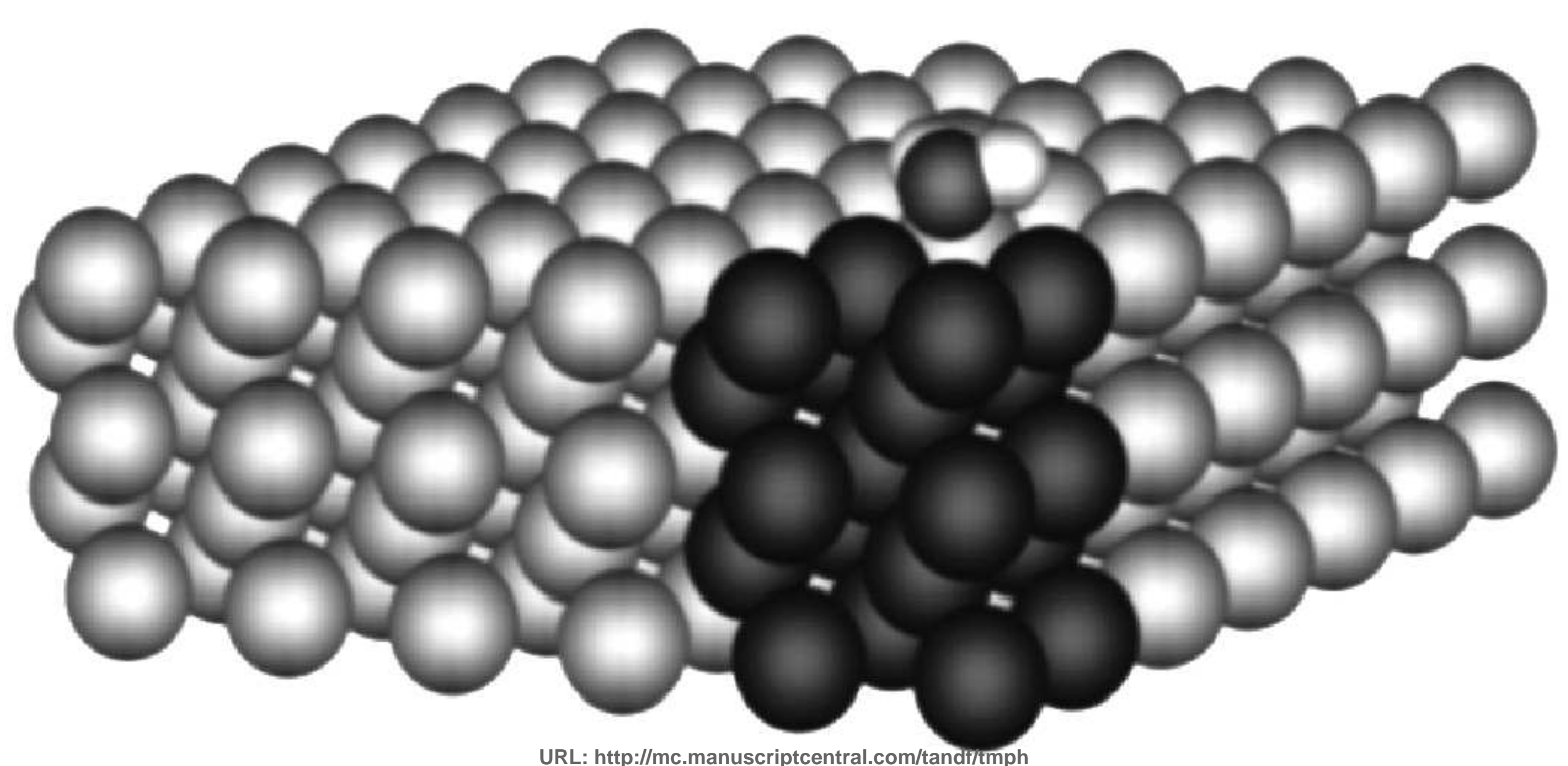

URL: http://mc.manuscriptcentral.com/tandiftmph 